\title{
OBTAINING OF CARBON NANOFIBERS BASED ON POLYACRYLONITRILE BY THE METHOD OF ELECTROSPINNING
}

\author{
Ospanali A.T., ${ }^{1,2}$, Kenzhegulov A.K. ${ }^{1,2}$, Zhumadilov B.E., ${ }^{1,2}$, Suyundykova G.S., ${ }^{1,2}$, \\ Medyanova B.S. ${ }^{1,2}$, Partizan G., ${ }^{1,2}$, Aliev B.A. ${ }^{2}$
}

\author{
${ }^{1}$ Center of innovative technology at the Institute of Combustion Problems, Almaty ,Kazakhstan \\ 2al-Farabi Kazakh National University, Almaty ,Kazakhstan, os_aziza@mail.ru
}

\begin{abstract}
Since carbon nanofibers comprise a continuous reinforcing with high specific surface area, associated with the fact that they can be obtained at a low cost and in a large amount, they have shown to be advantageous compared to traditional carbon nanotubes. The main objective of this work is the processing of carbon nanofibers, using polyacrylonitrile (PAN) as a precursor, obtained by the electrospinning process via polymer solution, with subsequent use as a reinforcement in polymer composites. The paper reports the fabrication and characterization of PAN nanofibers by electrospinning and further development of the as-spun PAN nanofibers into carbon nanofibers. PAN nanofibers as a precursor of carbon nanofibers with diameters in the range of 100-500 $\mathrm{nm}$ were prepared by electrospinning of PAN/DMF solution. The aligned electrospun PAN nanofibers first stabilized in air at temperature $250^{\circ} \mathrm{C}$ for $1 \mathrm{hr}$. We also investigated several carbonization procedures by varying final carbonization temperatures in the range from $600-900^{\circ} \mathrm{C}$ in argon atmospheres and the carbon nanofibers were successfully obtained at $700^{\circ} \mathrm{C}$ and $1 \mathrm{hr}$. Morphologies of PAN, stabilized and carbonized nanofibers were investigated by scanning electron microscopy (SEM). The results obtained from SEM showed that the average diameter of the stabilized and carbonized PAN nanofibers was significantly reduced.
\end{abstract}

Keywords: electrospinning, nanofibers, polyacrylonitrile, dimethylformamide, scanning electron microscopy.

\section{Introduction}

Interest to nanofibers caused by the fact that the mechanical properties of materials such as tensile strength, tear strength, bending and compression, the elastic modulus increase with decreasing fiber diameter and reach a theoretical limit when reaching the nanoscale. This is due, on the one hand, to a decrease in the concentration of extended defects in the nanofiber, and on the other hand, to a change in the physical properties of the nanofiber material itself due to the contribution of the surface. This effect is valid for any nanomaterials, since the surface of any material is a special two-dimensional ordered state. In the case of polymer nanofibers, the dimensional effect can also be manifested in the volume properties as a result of additional interaction between the polymer molecules caused by their orientation, when the fiber diameter becomes comparable to the length of the molecule [1]. One of the methods for producing nanofibers is the formation of fibers from polymer solutions under the action of an electrostatic field. This method combines hardware simplicity, high performance and scalability of the process from the laboratory installation to the elements of the industrial conveyor [2-5].

The paper presents the results of investigations by scanning electron microscopy of nanofibers based on polyacrylonitrile (PAN) synthesized by the method of electrospinning.

\section{Experiment details}

The solution was prepared using PAN powder (Mw: 150000) and dimethylformamide. In order for the powder to be completely dissolved, the solutions were mixed together and thoroughly mixed 
on a magnetic stirrer for 30 minutes at a temperature of $80^{\circ} \mathrm{C}$. Figure 1 shows a diagram of the installation of electrospinning. The laboratory unit consists of a high-voltage source, a special syringe pump, a collector that can rotate and supply additional voltage for more efficient collection of fibers, a syringe holder and the syringe with a metal needle connected to a high-voltage source. A polymer solution is placed in the syringe, which is then pumped out at a controlled speed through the needle. When a high voltage is applied to the liquid needle, the liquid jet breaks off from the surface of the drop and collects on the collector in the form of fibers. A series of experiments was carried out in which the distance from the needle to the collector $(20 \mathrm{~cm})$ and the supplied voltage $(16 \mathrm{kV})$ remained constant, the experiment duration was 3 hours, and the ratio of PAN and DMF concentrations varied from $6 \%$ to $10 \%$ in $2 \%$ increments.

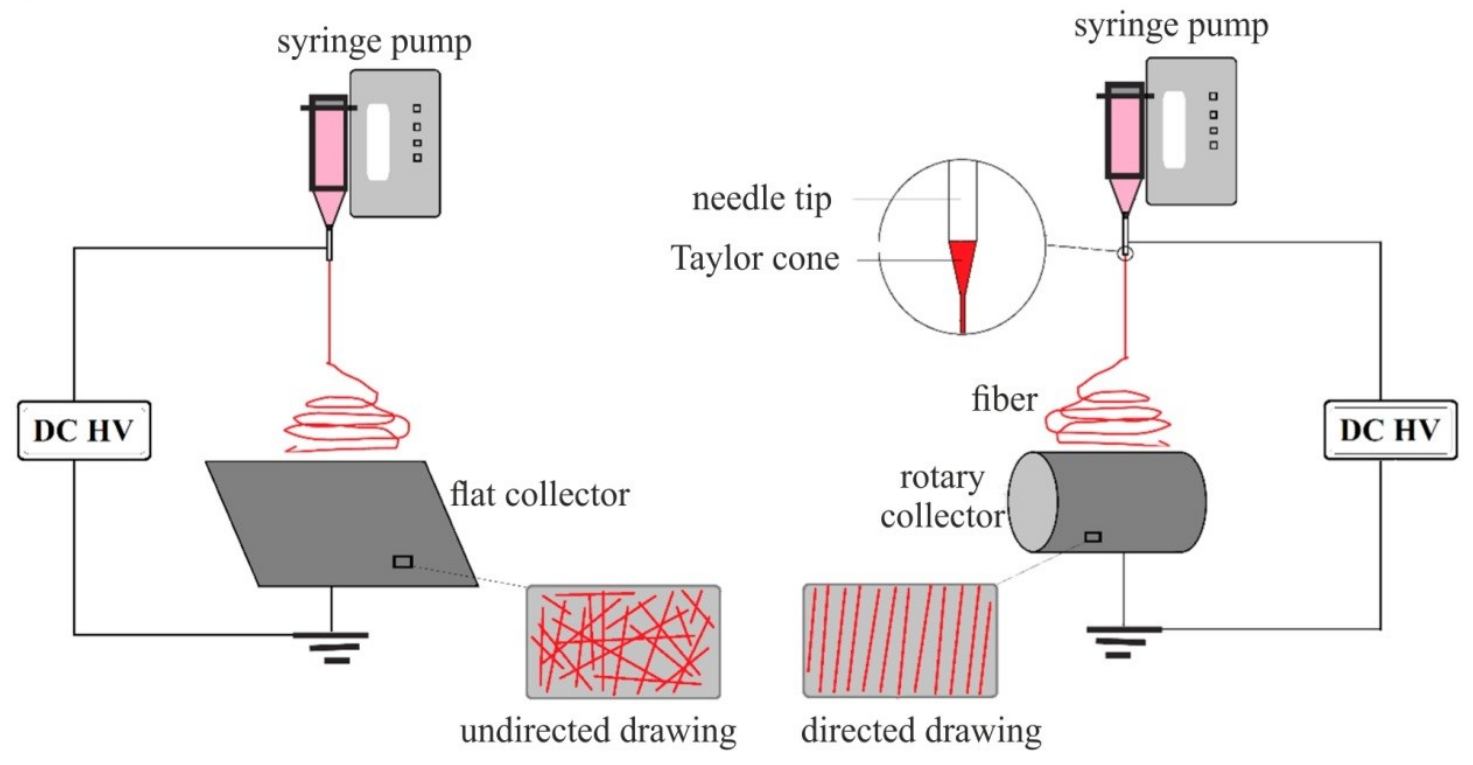

Fig.1. General scheme of the installation for the synthesis of nanofibers

In the second stage of the experiment, the synthesized fibers were dried in air before they were stabilized. Portions of samples were selected for heat treatment. For the stabilization process, the sample was placed in a horizontal three-zone tube furnace. The stabilization temperature was determined experimentally. The optimal stabilization temperature is $250{ }^{\circ} \mathrm{C}$. Further, the samples were carbonized at a temperature of $700^{\circ} \mathrm{C}$ in an argon atmosphere $(500 \mathrm{mbar})$. The carbonation temperature was also determined experimentally. The obtained samples were examined by the SEM method. The study of the samples was conducted at the National nanotechnology laboratory of open type with a microscope Quanta 3D 200i.

\section{Results and discussion}

To determine the effect of the parameters of various stages of the experiment on the diameters of nanofibers, histograms of the distribution of diameters were constructed. Figure 2 shows the picture, the SEM image and the histogram of diameters distribution of the synthesized carbon nanofibers a value of PAN concentration of $8 \%$. They are shown in different colors: white (after synthesis), brown (after the stabilization process at a temperature of $250^{\circ} \mathrm{C}$ ) and black (after the carbonization process at a temperature of $700^{\circ} \mathrm{C}$ ).

From the histogram data, it follows that the sample after synthesis is dominated by nanofibers with a diameter of $300-400 \mathrm{~nm}$, the average diameter of which is $369 \mathrm{~nm}$. After the stabilization process, the diameter of the fibers is reduced by a significant number the average diameter size is $298 \mathrm{~nm}$. It is also seen that after the carbonation process, the diameter of the fibers decreases. The 
average size of nanofibers after the carbonation process is $242 \mathrm{~nm}$. Histogram analysis shows that the distribution of nanofibers by diameter is Gaussian with a mean square deviation of $\sigma=25 \mathrm{~nm}$.
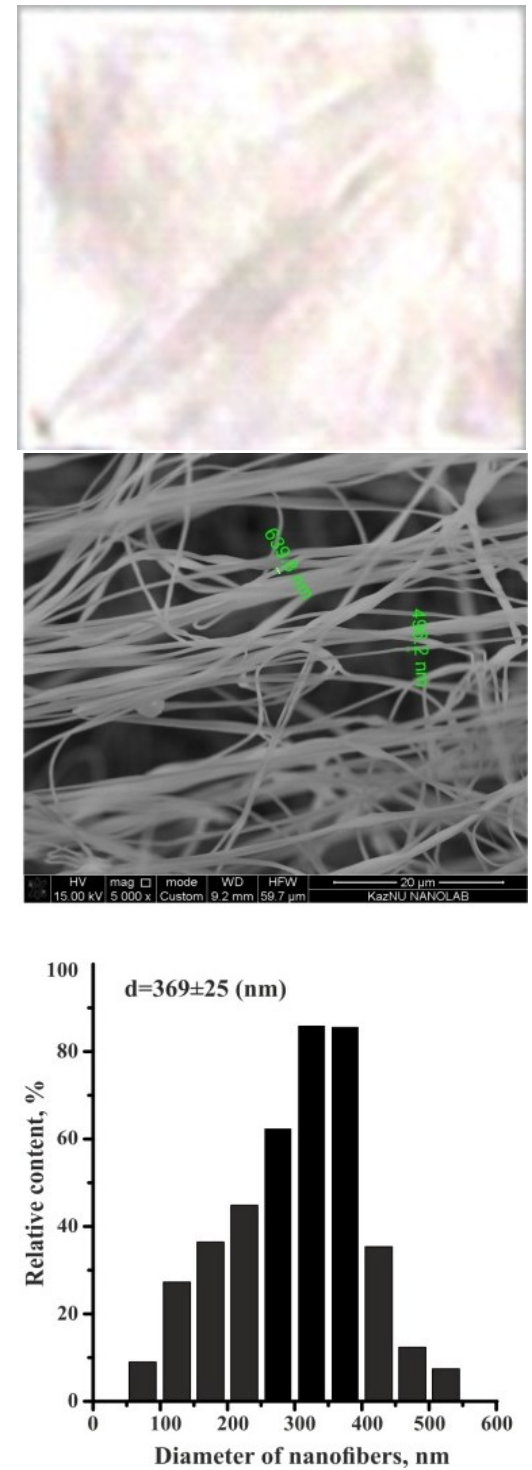

a
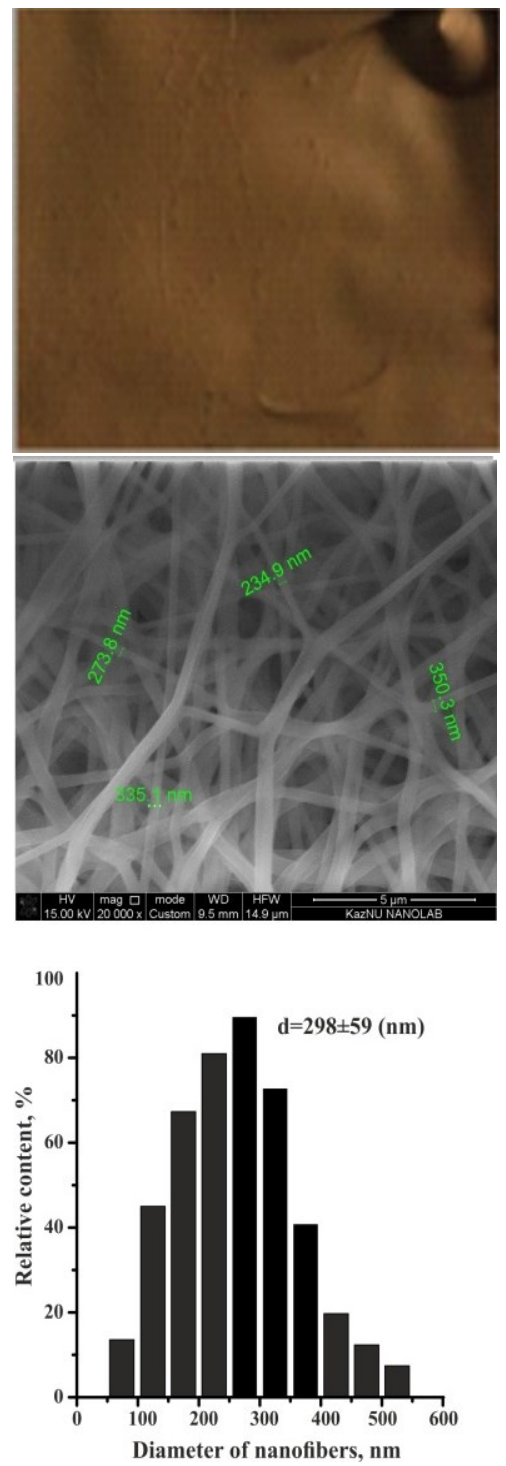

b
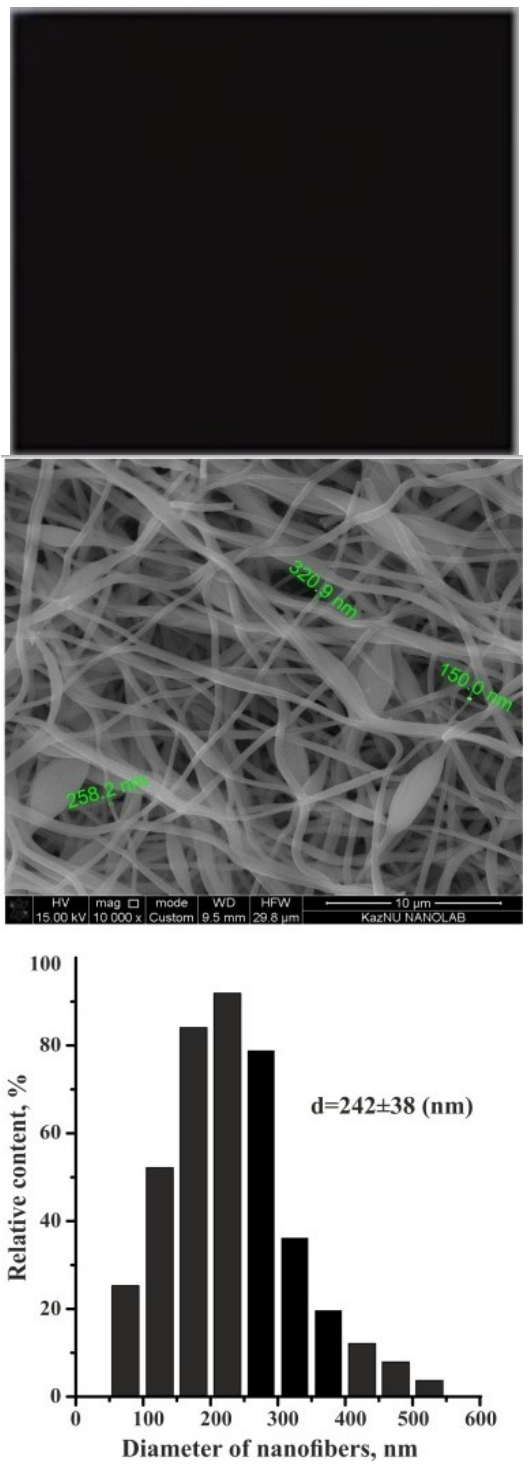

$\mathrm{c}$

Fig.2. Photographs, SEM images and histograms distribution of synthesized fiber diameters at the value of PAN $8 \%$ concentration: $\mathrm{a}$ - after synthesis, $\mathrm{b}$ - after the stabilization, $\mathrm{c}$ - after the carbonation

The results obtained from SEM and distribution of diameters showed that the average diameter of the stabilized and carbonized PAN nanofibers was significantly reduced. Analysis of literature data showed that the stabilization and carbonization process carried out significantly at high temperatures. Experimentally we determined the lower temperature limit for the stabilization and carbonization process. A series of experiments were carried out for check the repeatability of the results.

Figure 3 shows the SEM images after carbonation at a temperature of $700^{\circ} \mathrm{C}$ and a histogram of the distribution of nanofiber diameters at different values of PAN concentrations.

The results show that as the concentration of PAN decreases, the diameter of the nanofibers decreases. Thus, it was determined that the optimal concentration value for the synthesis of nanofibers with a minimum diameter is $6 \%$ 

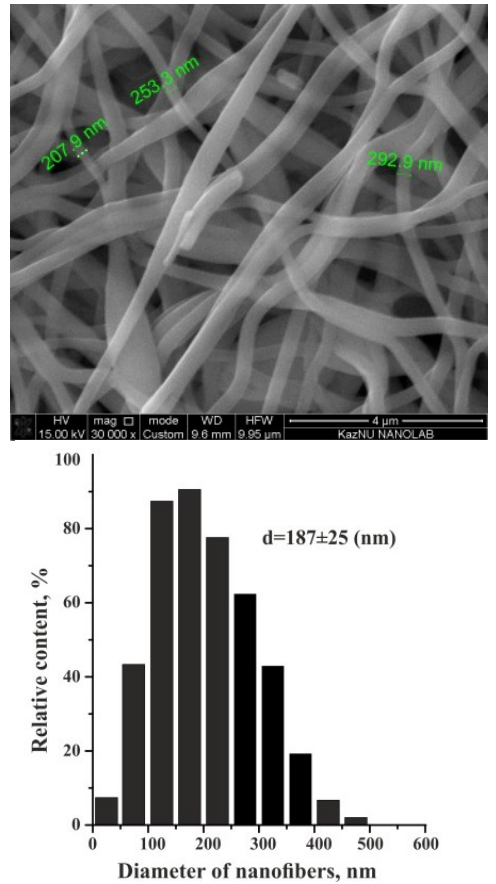

a
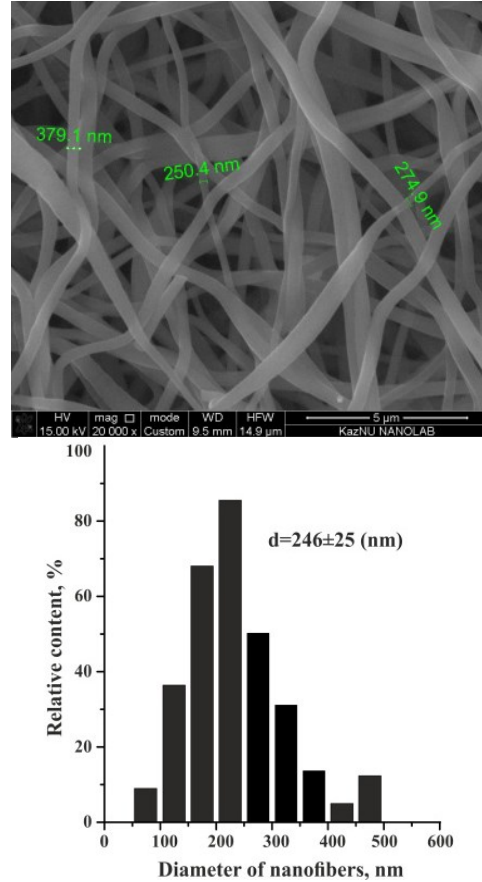

b
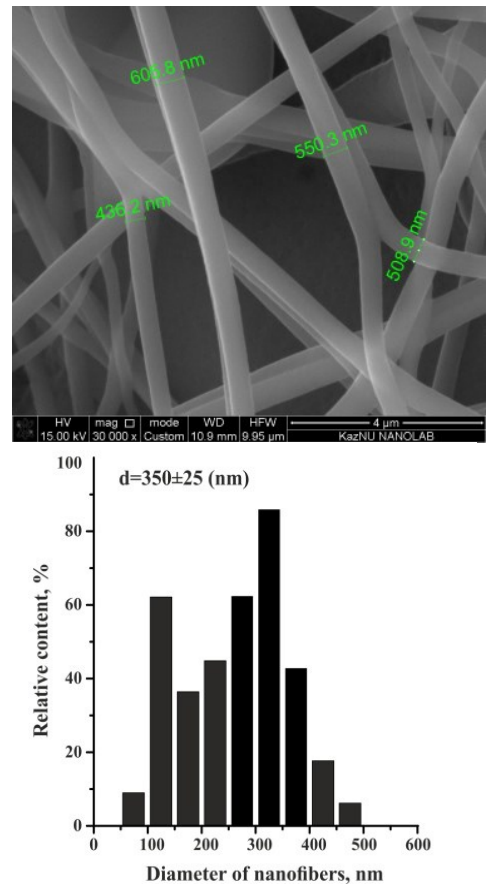

C

Fig.3. A histogram of the distribution of nanofiber diameters and SEM images after carbonation at different values of PAN concentrations (a) - 6\%; b) - 8\%; c) - 10\%)

\section{Conclusion}

During the research, trial experiments were conducted on the synthesis of carbon fibers by electrospinning based on PAN. The obtained samples were examined by the SEM method. According to the SEM analysis, the obtained samples have a homogeneous structure. The resulting nanofibers have a fairly large diameter spread from several tens to several hundred nanometers.

The optimal PAN concentration was determined for the synthesis of nanofibers with a minimum diameter. Further research will be aimed at determining the optimal combinations of technological parameters of synthesis, in order to obtain the necessary carbon nanofibers. For a more detailed analysis of the structure of the obtained structures, additional studies should be carried out using the methods of Raman scattering, X-ray fluorescence analysis, and transmission electron microscopy and electron diffraction.

\section{Acknowledgements}

The work was carried out with a partial financial support grant from the Science Committee of Ministry of Education and Science Republic of Kazakhstan. Grants No. AP05132865

\section{REFERENCES}

1 Li D., Wang Y., Xia Y. Electro spinning nanofibers as uniaxially aligned arrays and layer by-layer stacked films. Adv. Mater. 2004, Vol. 16, No. 4, pp. $361-366$.

2 Teo WE., Ramakrishna S. A review on electrospinning design and nanofibre assemblies. Nanotechnology, 2006, Vol. 89, pp. $395-407$.

3 Fang J., Zhang Li, Sutton D., Wang X., Lin T. Needleless Melt-Electrospinning of Polypropylene Nanofibres. Journal of Nanomaterials. 2012, Vol. 2012, pp. 1 - 9.

4 Chen D., Liu T., Zhou X., Chauhari W., Tjiu H. How Electrospinning Fabrication of High Strength and Toughness Polyimide Nanofiber Membranes Containing Multiwalled Carbon Nanotubes. Physical chemistry. 2009, Vol. 113, No. 29, pp. $9741-9748$.

5 Lyons J., Ko F. Melt Electrospinning of Polymers: A Review. The Polymer Science Monthly. 2005, Vol. 30, No. 6, pp. $170-178$. 